\title{
Prévalences des Myxosporidioses Chez Oreochromis niloticus Linné, 1758 (Cichlidae) au barrage réservoir de la Mapé (Adamaoua-Cameroun)
}

\author{
FONKWA Georges ${ }^{1,2^{\star}}$, TCHUINKAM Timoléon ${ }^{2}$, NANA TOWA Algrient ${ }^{1}$ et TCHOUMBOUE Joseph ${ }^{1}$ \\ 'Laboratoire d'Ichtyologie et d'Hydrobiologie Appliquée, Faculté d'Agronomie et des Sciences Agricoles, Université \\ de Dschang, B.P. 222, Dschang-Cameroun \\ 2 Laboratoire de Biologie et d'Écologie Appliquée, Unité de Recherche sur les Maladies Parasitaires et Infectieuses à \\ Transmission Vectorielle, Faculté des Sciences, Université de Dschang B.P. 67, Dschang-Cameroun \\ *Corresponding author's E-mail: fonkwageorges@gmail.com Tél. (+237) 674298924
}

Original submitted in on $11^{\text {th }}$ December 2017. Published online at www.m.elewa.org on $31^{\text {st }}$ March 2018 https://dx.doi.org/10.4314/jab.v123i1.2

\section{RÉSUMÉ}

Objectif : En vue de contribuer à une meilleure connaissance des myxosporidioses pour à terme élaborer des méthodes de lutte, 350 spécimens d'Oreochromis niloticus (tilapia du Nil) ont été échantillonnés de Mai 2016 à Mai 2017 au barrage de retenue de la Mapé (Adamaoua-Cameroun) et autopsiés.

Méthodologie et résultats: La collecte, conservation des poissons et l'identification des espèces de Myxosporidies ont été classiques. Douze espèces de Myxosporidies appartenant au genre Myxobolus ont été identifiées. Au total 159 poissons (45, 43\%) ont été parasités. Les espèces parasites ont été rares (Prévalence $<10 \%$ ) à l'exception de Myxobolus camerounensis, M. israelensis, M. tilapiae et M. brachysporus qui ont été intermédiaires $(10 \% \leq$ Prévalence $\leq 50 \%)$. La prévalence d'infestation a varié significativement entre les espèces parasites. La prévalence la plus élevée $(15,14 \%)$ et la plus faible $(0,86 \%)$ a été observée chez Myxobolus tilapiae et Myxobolus pharyngeus respectivement. Les poissons âgés ont été les plus infestés et les mâles plus parasités que les femelles toutefois, sans différence significative. Au total 10 organes ont été infestés. Indépendamment de l'espèce parasite, les reins ont été significativement les plus parasités $(36,57 \%)$ et ont hébergé 11 espèces parasites. M. camerounensis a montré un large spectre d'infestation des organes. La prévalence a été significativement plus élevée en saison sèche $(52,94 \%)$ qu'en saison de pluie $(39,59 \%)$. Au total, $59,94 \%$ de poissons parasités ont été poly infestés pour 5 catégories d'infestations multiples $(2 ; 3$; $4 ; 5$ et 6 espèces). La prévalence des associations d'infestations a baissé avec le nombre d'espèces parasites associées.

Conclusion et application: Les prévalences de myxosporidioses chez 0 . niloticus ont été affectées par les facteurs endogènes et les saisons. Les données permettent de développer des stratégies de lutte afin de booster la production en poisson.

Mots clés : Myxosporidies, Prévalence, Oreochromis niloticus, Barrage, Mapé, Cameroun 
Fonkwa et al., J. Appl. Biosci. 2018 Prévalences des Myxosporidioses Chez Oreochromis niloticus Linné, 1758 (Cichlidae) au barrage réservoir de la Mapé (Adamaoua-Cameroun)

Prevalence of Myxosporidiosis in Oreochromis niloticus Linnaeus, 1758 (Cichlidae) at Mapé reservoir dam (Adamawa-Cameroon)

Objectives: in order to contribute to a better understanding of Myxosporean infections to develop control strategies, 350 Oreochromis niloticus (Nile tilapia) specimens were sampled from May 2016 to May 2017 from the Mapé dam (Adamawa-Cameroon).

Methodology and results: Classical methods were used for fish's sampling, conservation and Myxosporean species identification. An overall total of 12 Myxosporean species belonging to the genus Myxobolus were identified. A total of 159 fish (45.43\%) were infected. Parasite species were scarce (Prevalence $<10 \%$ ) apart from Myxobolus camerounensis, M. israelensis, M. tilapiae and M. brachysporus which were intermediate $(10 \%$ $\leq$ Prevalence $\leq 50 \%)$. The Prevalence differed significantly between parasite species. The higher $(15.14 \%)$ and lower $(0.86 \%)$ prevalence was recorded for Myxobolus tilapiae and Myxobolus pharyngeus respectively. Moreover, older fish were the most infected and males were more parasitized than females with no significant difference. Out of 10 infected organs, kidneys were the most parasitized (36. 57\%) and harbored 11 parasite species. A broad spectrum of target organs was noticed for $M$. camerounensis. The prevalence was significantly higher in the dry season (52.94\%) than the rainy season (39.59\%). At total, $59.94 \%$ of infected fish were polyinfected and 5 types of polyinfections ( $2 ; 3 ; 4 ; 5$ and 6 species) were recorded. As, the number of combined species increased, the prevalence of polyinfection's categories dropped.

Conclusion and application: The prevalence of Myxosporidiosis in O. niloticus was influenced by endogenous factors and seasons. The recorded data are helpful for developing control strategies so as to boost the fish's production.

Keys words: Myxosporean, Prevalence, Oreochromis niloticus, dam, Mapé, Cameroon

\section{INTRODUCTION}

En Afrique, le boom démographique est à l'origine de l'augmentation de la demande en produits alimentaires en général et halieutiques en particulier. Parmi les ressources halieutiques, le poisson fournit environ $51 \%$ de protéines animales dans l'alimentation (FAO, 2016). Au Cameroun, la production nationale en poissons n'est que de 180 $\mathrm{x} 10^{3}$ tonnes par an alors que la demande est estimée à $400 \times 10^{3}$ tonnes. C'est pourquoi $250 \times 10^{3}$ tonnes de poissons supplémentaires représentant 100 milliards de FCFA de dépense sont importées chaque année (MINEPIA, 2015) alors que ce pays dispose d'un grand potentiel hydrographique (4 millions d'ha de superficie totale) sous forme de plans d'eaux naturels et artificiels ainsi, constitue un immense reservoir en poissons. D'une superficie d'environ $550 \mathrm{~km}^{2}$, le barrage de retenue de la Mapé particulièrement a vu sa production annuelle en poissons baisser de $15 \%$ entre 1990 et 2015 (MINEPIA, 2015). Les raisons ne seraient pas seulement les changements climatiques (Renault et Guichard, 2007) et la surpêche, mais aussi les pathologies notamment parasitaires parmi lesquelles les Myxosporidies (Abakar, 2006). Ces dernières sont à effets très néfastes (Lom et Dyková, 2006). Elles affectent tous les organes aussi bien des poissons d'eaux douces que marines (Fomena, 1995). Les Myxosporidies non seulement affaiblissent et tuent les poissons, provoquent des pertes de production (Gbankoto et al., 2001; Feist et Longshaw, 2005), mais aussi interfèrent sur leurs performances de production telles que le gain pondéral (Longshaw et al., 2010) et la reproduction (Obiekezie et Okaeme,1990). En Afrique, les travaux sur les Myxosporidies portent plus sur la faunistique que la dynamique (Abakar, 2006) or il est important d'associer à l'étude faunistique la dynamique, c'est pour quoi l'objectif général du présent travail est de contribuer à l'augmentation des potentiels de production en poissons à travers une meilleure connaissance de leurs pathologies/myxosporidioses et des méthodes de lutte. Plus spécifiquement, il s'agit d'évaluer l'effet des facteurs endogènes (âge du poisson, sexe, organes) et exogènes (saisons) sur les prévalences des myxosporidioses chez 0 . niloticus. 

1758 (Cichlidae) au barrage réservoir de la Mapé (Adamaoua-Cameroun)

\section{MATÉRIEL ET MÉTHODES}

Zone d'étude et sites d'échantillonnage : La zone d'étude (Figure 1) est l'arrondissement de Bankim situé entre $6^{\circ} 00^{\prime}-6^{\circ} 20^{\prime} \mathrm{LN}$ et $11^{\circ} 20^{\prime}-11^{\circ} 40^{\prime} \mathrm{LE}$, Département de Mayo-Banyo, Région administrative de l'AdamaouaCameroun (LN : $9^{\circ}-16^{\circ}$, LE : $2^{\circ}-13^{\circ}$, Afrique Centrale). L'altitude moyenne est de $724 \mathrm{~m}$. Les sols sont argilosableux, latéritiques et ferralitiques. Le climat est de type tropical soudano-guinéen à deux saisons : une saison pluvieuse qui va de mi-Mars à mi- Novembre et une saison sèche de mi-Novembre à mi- Mars. La pluviométrie annuelle moyenne varie entre 1500 et 2000 $\mathrm{mm}$ et la température moyenne est de $23^{\circ} \mathrm{C}$ (Olivry, 1986). La figure 1 montre les sites d'échantillonnage au barrage de retenue de la Mapé ainsi que les principaux cours d'eaux. Les poissons ont été collectés mensuellement auprès des pêcheurs de Mai 2016 à Mai 2017. La pêche se faisait grâce aux filets éperviers et maillants $(1 \mathrm{~cm} X 1 \mathrm{~cm})$, des lignes et nasses. Sur le terrain, les poissons ainsi obtenus ont été immergés dans une solution de formol $10 \%$, puis transportés au Laboratoire. Au total 350 poissons (219 mâles et 131 femelles) ont été échantillonnés. Les longueurs standards ont varié de 51 à $240 \mathrm{~mm}$ avec une moyenne de 97,13 $\mathrm{mm}$. Sur la base des ces longueurs, les poissons ont été repartis en 3 classes de tailles d'amplitude $50 \mathrm{~mm}$. La masse a fluctué entre 4, 88 et 152, $39 \mathrm{~g}$ avec une moyenne de 31,94g.

Identification des Myxosporidies: Au laboratoire, chaque poisson est examiné selon la méthode utilisée par Abakar (2006). A cet effet, les longueurs standard et totale ont été mesurées à l'aide d'un ichtyomètre de précision 0,1 millimètre. La masse a été pesée à l'aide d'une balance électronique de marque Sartorius, de portée $2200 \mathrm{~g}$ et au centième de gramme près. Les organes externes (yeux, peau, nageoires, opercules et écailles) ont été examinés à l'œil nu pour déceler la présence éventuelle des kystes macroscopiques, puis à la loupe binoculaire de marque Motic et au grossissement 10X. Après dissection de l'animal, le sexe a été déterminé, tous les organes internes (branchies, rate, foie, intestins, cerveau, reins, gonades, cœur et vésicule biliaire) ont été prélevés, placés dans des boites de pétri contenant de l'eau de robinet, examinés à l'œil nu puis à la loupe à la loupe binoculaire. Les kystes parasitaires trouvés ont été dénombrés puis écrasés entre lame et lamelles dans une goutte d'eau distillée et leurs contenus ont été identifiés au microscope électrique au grossissement 1000X. Les frottis des reins, de la rate et foie ont été également confectionnés et examinés. Les spores fixées au méthanol puis colorées au MayGrünwald-Giemsa ont été photographiées à l'aide d'un appareil photographique numérique de marque Canon Ixus. Les différentes espèces de myxosporidies ont été identifiées selon les recommandations de Lom et Arthur (1989).

Indice épidémiologique étudié : La prévalence $(\mathrm{Pr})$ d'infestation exprimée en pourcentage est définie comme étant le rapport du nombre de poissons infestés par une espèce parasite donnée par le nombre examiné (Bush et al., 1997). L'analyse du statut de chaque parasite a été faite selon Valtonen et al. (1997). Ainsi, les espèces sont qualifiées de fréquentes ou principales ( $\mathrm{Pr}>50 \%$ ), secondaires ou intermédiaires $(10 \% \leq \operatorname{Pr} \leq 50 \%)$, rares ou satellites $(\operatorname{Pr}<10 \%)$.

Analyses statistiques : Le test de Chi - deux $\left(\mathrm{X}^{2}\right)$ a été utilisé pour comparer les prévalences d'infestations. Le seuil de signification $5 \%$ a été retenu et le logiciel SPSS version 20.0 a servi aux analyses. 


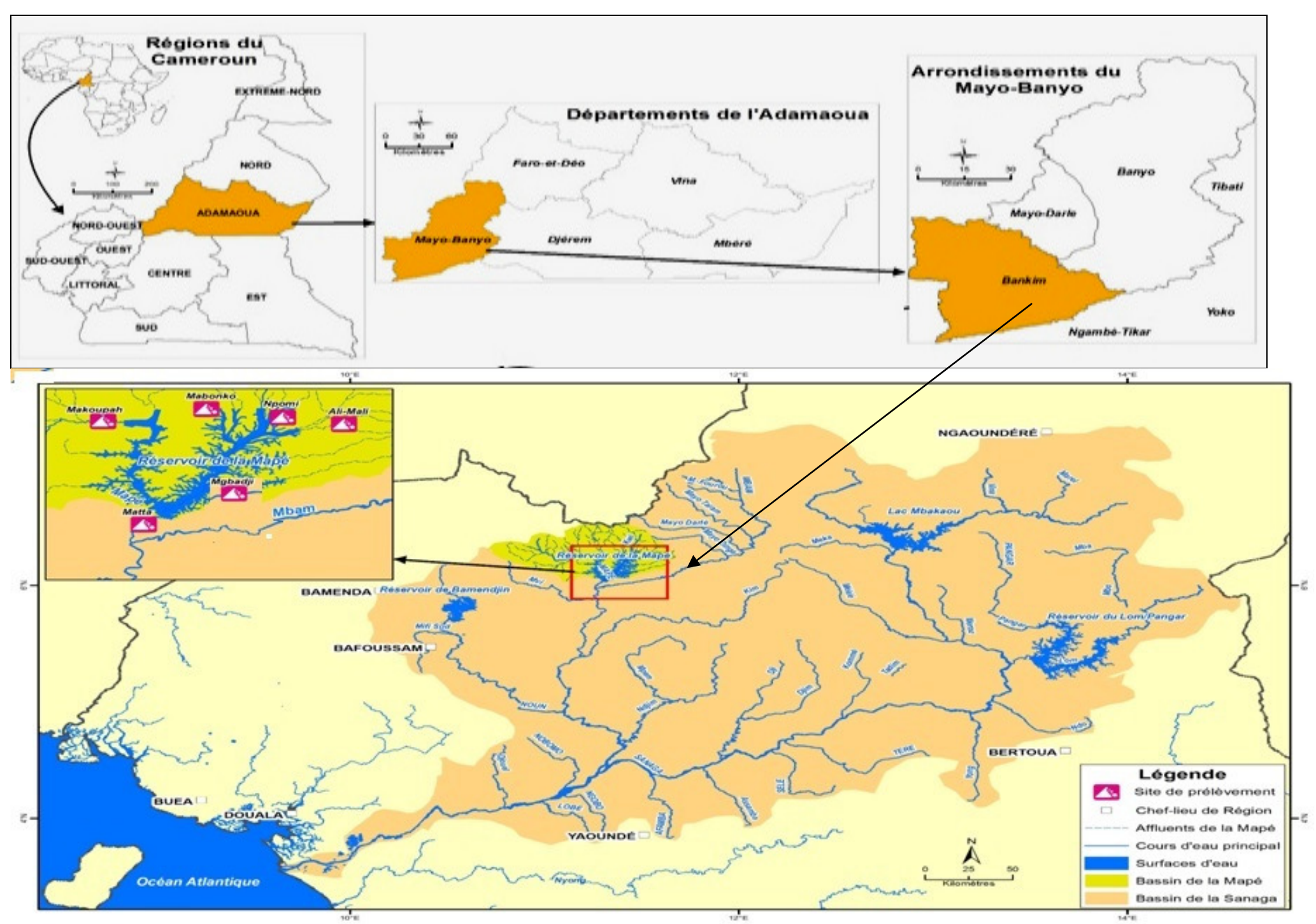

Figure 1 : Localisation géographique de la zone d'étude, des sites d'échantillonnage et illustration du bassin hydrographique de la Sanaga. 

1758 (Cichlidae) au barrage réservoir de la Mapé (Adamaoua-Cameroun)

\section{RÉSULTATS}

Prévalences des espèces de Myxosporidies : La figure 2 montre que 12 espèces de myxosporidies appartenant toutes au genre Myxobolus ont été recensées. Les prévalences des espèces de myxosporidies telles qu'illustrées par la figure 3 sont de $45,43 \%$ indépendamment de l'espèce parasite. Les espèces parasites sont rares à l'exception de Myxobolus camerounensis, $M$. israelensis, $M$. tilapiae et $M$. brachysporus qui sont intermédiaires. Les prévalences d'infestations ont varié très significativement entre les espèces parasites $\left(X^{2}=140,40 ; P=0,001\right)$. Les prévalences les plus élevées $\left(X^{2}=140,40 ; P<0,001\right)$ sont observées chez Myxobolus tilapiae $(15,14 \%)$ suivi de $M$. brachysporus $(12,29 \%)$ et la plus faible chez Myxobolus pharyngeus.

Prévalences des espèces parasites en fonction des classes de tailles: Les prévalences des espèces parasites en fonction des classes de tailles sont résumées dans le tableau 1. II en ressort que les poissons sont parasités dans toutes les classes considérées. Indépendamment de l'espèce parasite, les prévalences varient significativement $\left(X^{2}=6,20 ; P<0,05\right)$ entre les classes de tailles. La plus faible prévalence $(40,51 \%)$ est notée dans la classe [50 - 100] contrairement aux deux dernières classes qui montrent des prévalences élevées et comparables $(54,55 \%)$. La comparaison des classes de tailles en fonction du nombre d'espèces parasites hébergées montre que toutes les 12 espèces parasites sont représentées dans les deux premières classes contrairement aux poissons de tailles supérieures à $150 \mathrm{~mm}$ qui ne sont infestés que par 8 espèces de myxosporidies. A espèce parasite égale, seules les prévalences d'infestations par M. tilapiae ont très significativement varié entre les trois classes de tailles $\left(X^{2}=9,30 ; P<0,001\right)$, ce parasite étant plus prévalent $(36,36 \%)$ dans la plus grande classe de taille. En comparant les prévalences des espèces parasites à l'intérieur des classes de tailles, seules les bornes [50 100] et] 100 - 150] montrent des prévalences très significativement différentes $(P<0,001)$ entre les espèces parasites.

Prévalences d'infestations en fonction du sexe des hôtes: Les prévalences d'infestations en fonction du sexe des hôtes ressorties par la figure 4 montre que, aussi bien les poissons mâles que femelles sont infestés. Indépendamment de l'espèce parasite, les mâles sont plus parasités que les femelles, cependant sans différence significative $\left(X^{2}=0,61 ; P=0,436\right)$. A sexe égal, toutes les espèces parasites sont communes aux poissons mâles et femelles à l'exception de $M$. kainjiae spécifique aux femelles. Quel que soit le sexe considéré, les prévalences varient hautement et significativement $(P$ $<0,001$ ) entre les espèces parasites. Lorsqu'on compare les espèces parasites par rapport à leurs affinités aux sexes des hôtes, il apparaît que, contrairement à $\mathrm{M}$. Kainjiae, $M$. tchadanayei, $M$. pharyngeus, $M$. pseudodispar et M. brachysporus, les 7 autres parasites infestent plus les mâles que les femelles. Cependant aucune différence significative $(P>0,05)$ n'est observée entre les prévalences; seul $M$. kainjiae est significativement plus prévalent chez les femelles que les mâles $\left(X^{2}=26,20 ; P<0,001\right)$.

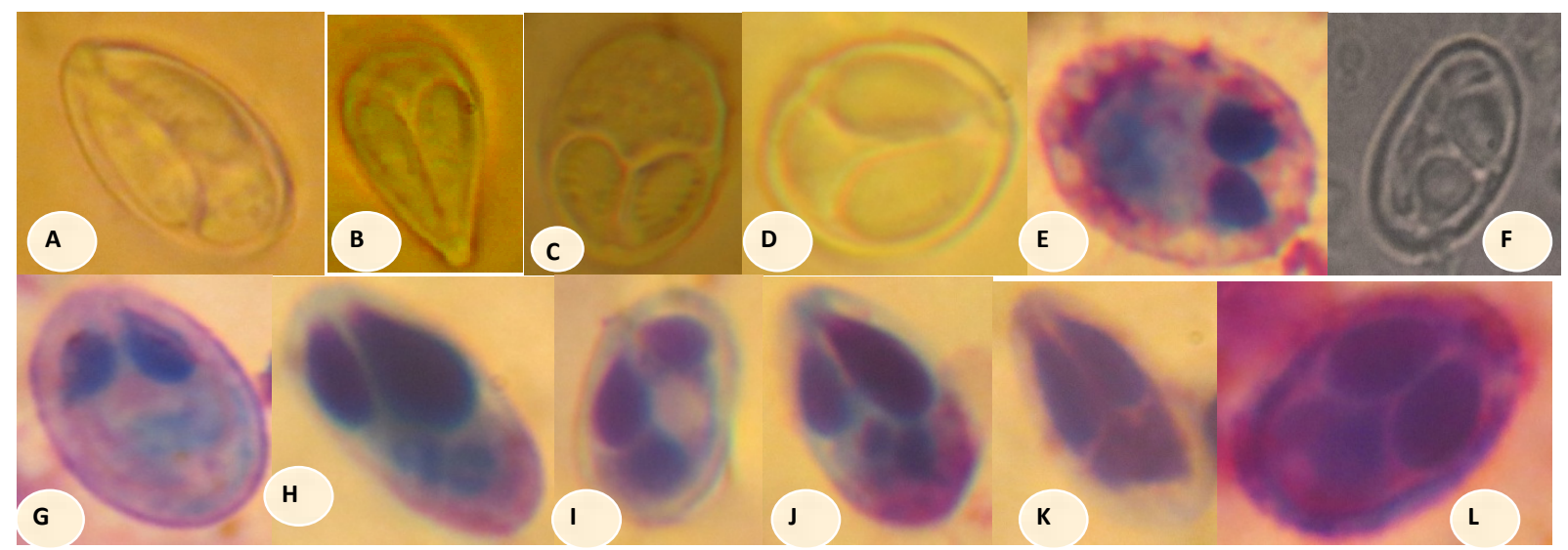

A :Myxobolus israelensis (x1600); B : Myxobolus heterosporus (x 1600); C : Myxobolus camerounensis (x 1500) ; D: Myxobolus agolus (x1600); E: Myxobolus kainjiae (x 1800); F: Myxobolus brachysporus (x 1500); G : Myxobolus tilapiae (x 1500); H : Myxobolus mapei (x 1200); I: Myxobolus pseudodispar (x 1200); J: Myxobolus tchadanayei (x 1600); K: Myxobolus pharyngeus (x1600); L :Myxobolus ellipsoides (x 1600)

Figure 2 : Microphotographies des spores des différentes espèces de Myxosporidies étudiées 


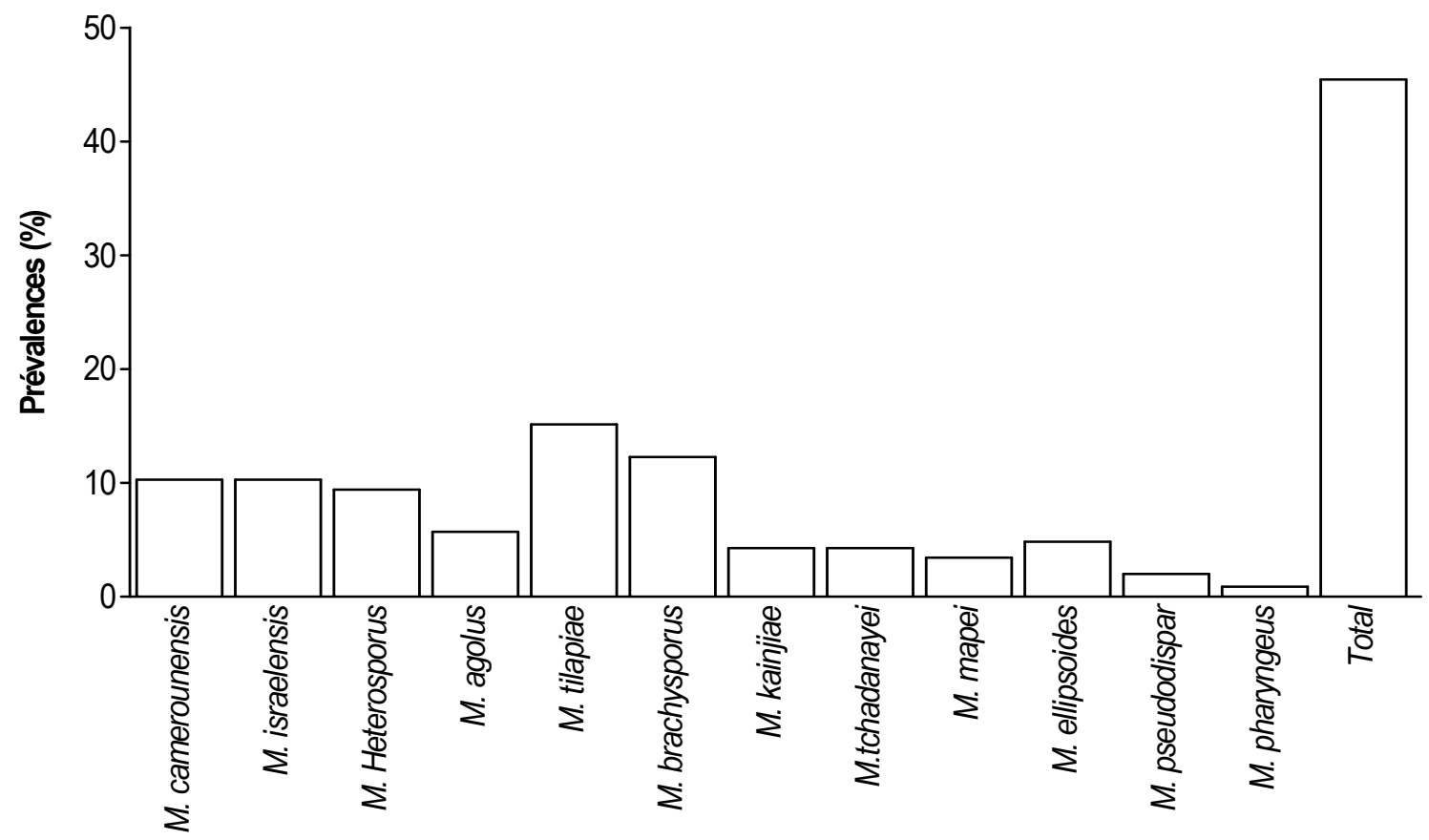

\section{Espèces parasites}

Figure 3 : Prévalences des espèces de Myxosporidies

Tableau 1 : Prévalences des espèces parasites en fonction des classes de tailles

\begin{tabular}{|c|c|c|c|c|c|}
\hline \multirow[b]{2}{*}{ Espèces parasites } & \multicolumn{3}{|c|}{ Classes de tailles (mm) } & \multirow[b]{2}{*}{$X^{2}$} & \multirow[b]{2}{*}{$P$} \\
\hline & $\begin{array}{c}{[50-100]} \\
229^{*}\end{array}$ & $\begin{array}{l}\text { ] } 100-150] \\
110^{*}\end{array}$ & $\begin{array}{r}>150 \\
11^{*}\end{array}$ & & \\
\hline M. camerounensis & 9,61 & 12,73 & 0,00 & 2,10 & 0,352 \\
\hline M. israelensis & 9,17 & 10,91 & 27,27 & 3,80 & 0,150 \\
\hline M. heterosporus & 17,86 & 13,64 & 0,00 & 4,10 & 0,130 \\
\hline M. agolus & 5,68 & 5,45 & 9,09 & 0,25 & 0,883 \\
\hline M. tilapiae & 11,35 & 20,91 & 36,36 & 9,30 & 0,01 \\
\hline M. brachysporus & 9,17 & 19,09 & 9,09 & 6,90 & 0,031 \\
\hline M. kainjiae & 3,06 & 0,64 & 9,09 & 2,60 & 0,270 \\
\hline M. tchadanayei & 4,37 & 3,64 & 9,09 & 0,74 & 0,692 \\
\hline M. mapei & 2,62 & 4,55 & 9,09 & 1,93 & 0,381 \\
\hline M. ellipsoides & 4,37 & 5,45 & 9,09 & 0,63 & 0,730 \\
\hline M. pseudodispar & 1,75 & 2,73 & 0,00 & 0,60 & 0,742 \\
\hline M. pharyngeus & 0,44 & 21,82 & 0,00 & 1,74 & 0,419 \\
\hline Total & 40,51 & 54,55 & 54,55 & 6,20 & $<0,05$ \\
\hline $\mathrm{X}^{2}$ & 58,50 & 61,39 & 17,32 & & \\
\hline$P$ & $<0,001$ & $<0,001$ & 0,099 & & \\
\hline
\end{tabular}



1758 (Cichlidae) au barrage réservoir de la Mapé (Adamaoua-Cameroun)

Prévalences des espèces parasites en fonction des organes: Les prévalences des espèces parasites en fonction des organes (Tableau 2) indiquent un total de 10 organes parasités. Indépendamment de l'espèce parasite, les reins sont significativement les plus parasités $\left(X^{2}=719,60 ; P<0,001 ; \operatorname{Pr}=36,57 \%\right)$. Par ailleurs, la plus faible prévalence est notée au niveau des intestins. La comparaison de la richesse des organes en espèces parasites montre que les reins, le foie et la rate hébergent plus d'espèces de myxosporidies que autres organes, soit respectivement $11 ; 7$ et 4 espèces. Les nageoires et les ovaires n'étant parasités que par une seule espèce. A l'exception du foie, de la rate et des intestins, les prévalences d'infestations des organes sont hautement et significativement différentes $(P<0,001)$ entre les espèces parasites. A espèce parasite égal, le plus large spectre d'infestation est observé chez M. camerounensis infestant 6 organes sur 10 avec des prévalences allant de $0,86 \%$ dans les branchies à $3,14 \%$ au niveau des reins. $A$ l'opposé M. pharyngeus, M. pseudodispar et M. kainjiae infestent chacun un seul organe. Quel que soit le parasite pris en compte, les prévalences ne sont pas comparables $(P<0,05)$ entre les organes

Prévalences d'infestations en fonction des saisons : Les prévalences d'infestations en fonction des saisons ainsi illustrées par la figure 5 montre que les poissons sont infestés pendant les deux saisons. Indépendamment de l'espèce parasite, les prévalences sont significativement plus élevées en saison sèche qu'en saison de pluie $\left(X^{2}=6,19 ; P=0,013\right)$. Quelle que soit la saison, les prévalences des espèces parasites varient très significativement $(P<0,001)$. Elles oscillent entre 0 (M. pharyngeus) et 12, 18\% (M. tilapiae), puis de 0,65 (M. pseudodispar) à 18, 95\% (M. tilapiae) respectivementen saison de pluie et en saison sèche. Lorsqu'on compare les prévalences d'occurrence des parasites en fonction des saisons (Figure 5), il ressort que toutes les espèces parasites apparaissent pendant les deux saisons à l'exception de $M$. pharygeus qui n'est présent qu'en saison sèche $\left(X^{2}=3,90 ; P=0,048\right)$. Seuls $M$. heterosporus $\left(X^{2}=0,28 ; P=0,599\right)$ et $M$. pseudodispar $\left(X^{2}=2,51 ; P=0,113\right)$ sont les parasites plus présents en saison de pluie qu'en saison sèche.

Prévalences des types d'infestations: Les Prévalences des types d'infestations sont illustrées par la figure 6 . Il en ressort deux types d'infestations notamment les mono infestations (infestations simples) et les poly infestations (infestations multiples) dont les prévalences sont comparables $\left(X^{2}=0,07 ; P=0,787\right)$. Par ailleurs, 5 catégories de poly infestations sont observées précisément les infestations bi, tri, trétra, penta et hexa spécifiques. Les prévalences des infestations multiples décroissent très significativement avec le nombre d'espèces parasites associées ( $\left.X^{2}=140,40 ; P<0,001\right)$.

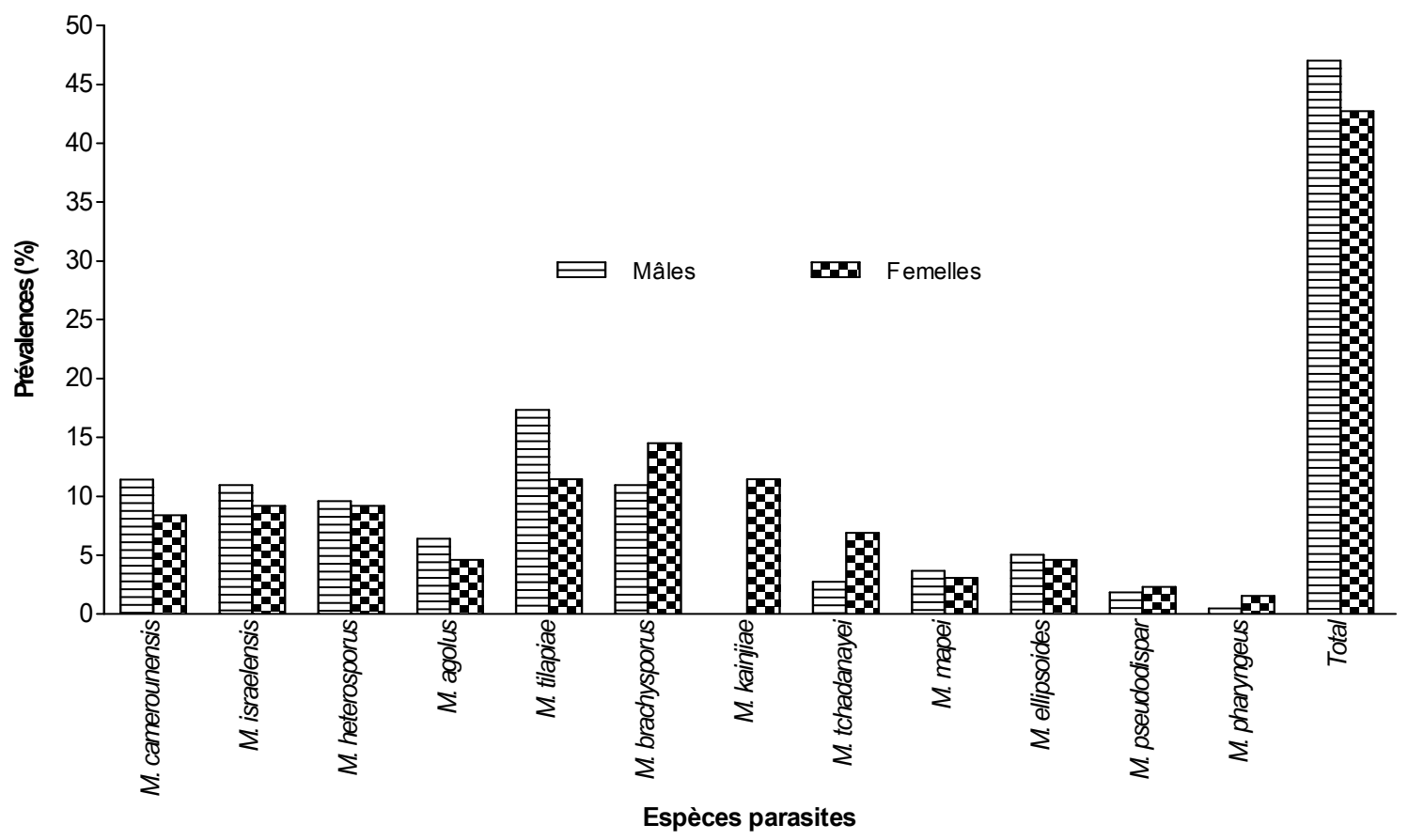

Figure 4: Prévalences d'infestations en fonction du sexe des hôtes 


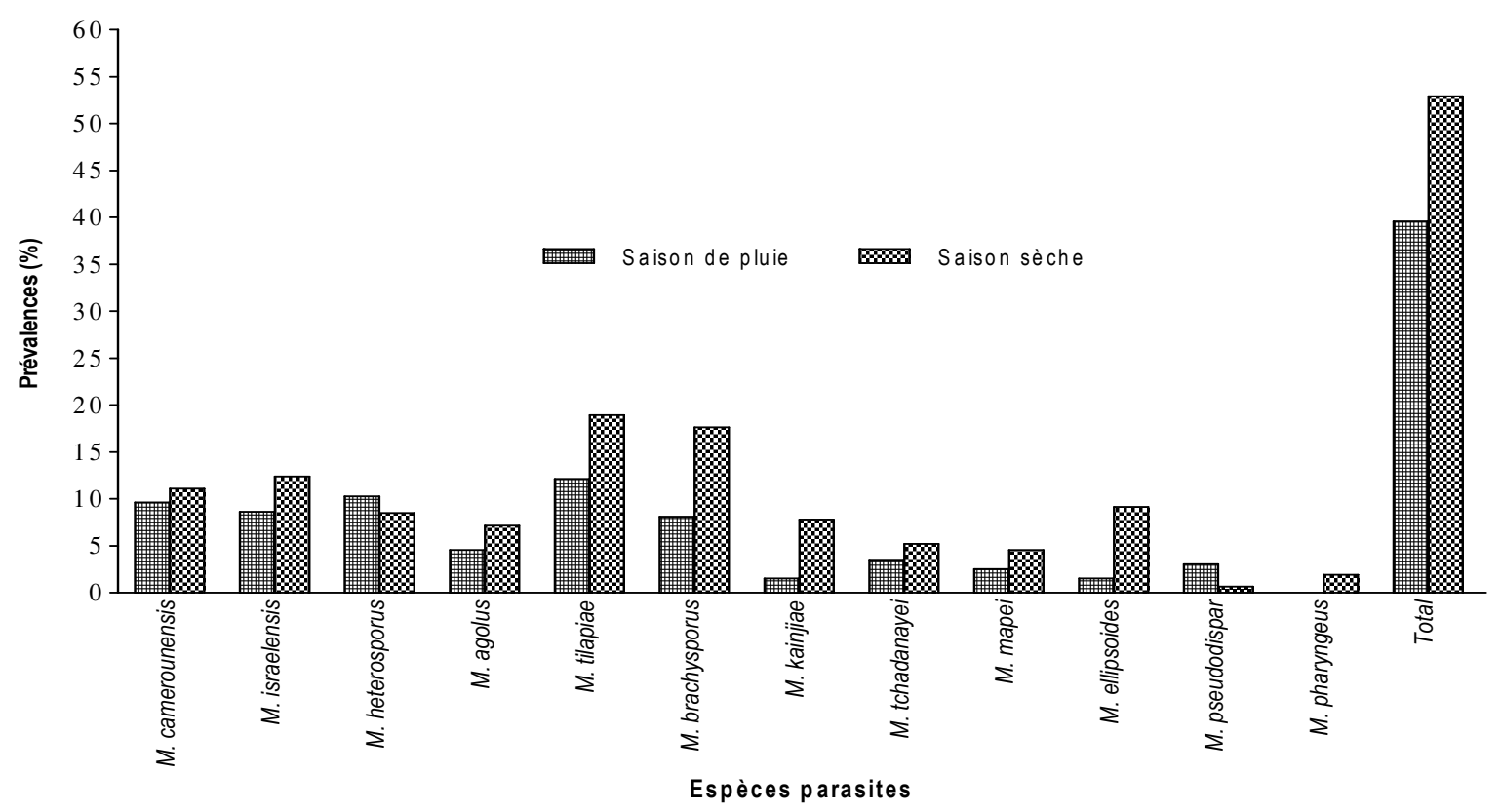

Figure 5 : Prévalences d'infestations en fonction des saisons

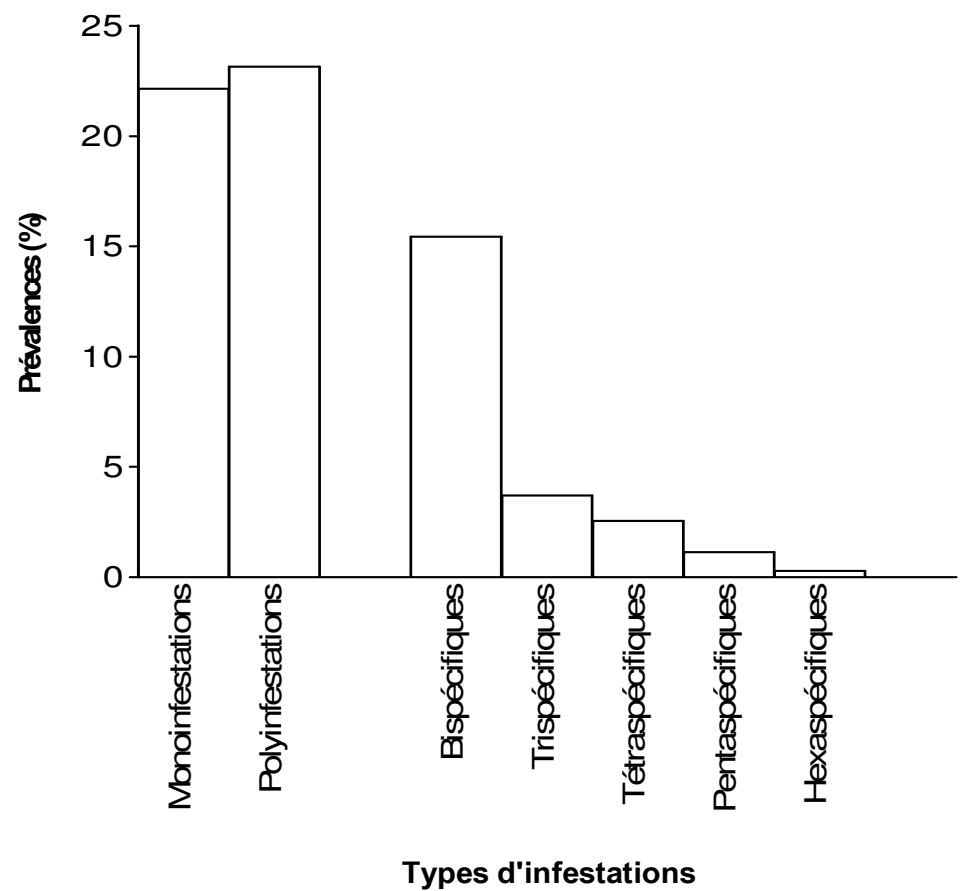

Figure 6 : Prévalences des types d'infestations 
Fonkwa et al., J. Appl. Biosci. 2018 Prévalences des Myxosporidioses Chez Oreochromis niloticus Linné, 1758 (Cichlidae) au barrage réservoir de la Mapé (Adamaoua-Cameroun)

Tableau 2 : Prévalences des espèces parasites en fonction des organes

\begin{tabular}{|c|c|c|c|c|c|c|c|c|c|c|c|c|}
\hline \multirow{2}{*}{$\begin{array}{l}\text { Espèces } \\
\text { parasites }\end{array}$} & \multicolumn{8}{|c|}{ Organes parasités } & & & \multirow[t]{2}{*}{$x^{2}$} & \multirow[t]{2}{*}{$P$} \\
\hline & Yeux & Opercules & Peau & Nageoires & Branchies & Reins & Foie & Rate & Intestins & Ovaires & & \\
\hline $\begin{array}{l}\text { M. } \\
\text { camerounensis }\end{array}$ & 2,28 & 1,43 & 1,43 & 2,00 & 0,86 & 3,14 & 0,00 & 0,00 & 0,00 & 0,00 & 36,54 & $<0,001$ \\
\hline M. israelensis & 0,00 & 0,57 & 0,29 & 0,00 & 0,00 & 8,57 & 0,00 & 0,86 & 0,00 & 0,00 & 227,60 & $<0,001$ \\
\hline M. heterosporus & 0,57 & 0,00 & 0,00 & 0,00 & 0,00 & 8,00 & 0,57 & 0,57 & 0,00 & 0,00 & 209,30 & $<0,001$ \\
\hline M. agolus & 0,00 & 0,00 & 0,00 & 0,00 & 0,00 & 5,43 & 0,29 & 0,00 & 0,00 & 0,00 & 161,90 & $<0,001$ \\
\hline M. tilapiae & 0,00 & 0,00 & 0,29 & 0,00 & 0,57 & 14,00 & 0,57 & 0,29 & 0,00 & 0,00 & 398,10 & $<0,001$ \\
\hline M. brachysporus & 0,00 & 0,00 & 0,00 & 0,00 & 0,00 & 11,14 & 0,57 & 0,29 & 0,57 & 0,00 & 316,0 & $<0,001$ \\
\hline M. kainjiae & 0,00 & 0,00 & 0,00 & 0,00 & 0,00 & 0,00 & 0,00 & 0,00 & 0,00 & 4,29 & 135,60 & $<0,001$ \\
\hline M. tchadanayei & 0,00 & 0,00 & 0,29 & 0,00 & 0,00 & 3,14 & 0,29 & 0,00 & 0,29 & 0,00 & 69,97 & $<0,001$ \\
\hline M. mapei & 0,00 & 0,00 & 0,00 & 0,00 & 0,00 & 2,86 & 0,57 & 0,00 & 0,00 & 0,00 & 74,92 & $<0,001$ \\
\hline M. ellipsoides & 0,00 & 0,00 & 0,00 & 0,00 & 0,00 & 4,57 & 0,29 & 0,00 & 0,00 & 0,00 & 134,80 & $<0,001$ \\
\hline M. pseudodispar & 0,00 & 0,00 & 0,00 & 0,00 & 0,00 & 2,00 & 0,00 & 0,00 & 0,00 & 0,00 & 63,13 & $<0,001$ \\
\hline M. pharyngeus & 0,00 & 0,00 & 0,00 & 0,00 & 0,00 & 0,85 & 0,00 & 0,00 & 0,00 & 0,00 & 27,02 & $<0,01$ \\
\hline Total & 2,85 & 2,00 & 2,29 & 2,00 & 1,43 & 36,57 & 2,00 & 1,43 & 0,86 & 4,29 & 719,60 & $<0,001$ \\
\hline $\mathrm{X}^{2}$ & 54,62 & 37,40 & 32,40 & 88,17 & 26,02 & 140,90 & 9,75 & 18,75 & 10,00 & 165,60 & & \\
\hline$P$ & $<0,001$ & $<0,001$ & $<0,001$ & $<0,001$ & $<0,01$ & $<0,001$ & 0,553 & 0,066 & 0,530 & $<0,001$ & & \\
\hline
\end{tabular}


Prévalences des types d'infestations en fonction des saisons : Les prévalences des types d'infestations en fonction des saisons telles que mises en évidence par la figure 7 montre la présence des mono et poly infestations pendant les deux saisons. La prévalence des mono infestations est plus élevée $\left(X^{2}=0,26 ; P=0,613\right)$ en saison de pluie que celle des poly infestations. A l'inverse, en saison sèche, les poly infestations sont les plus représentées $\left(X^{2}=0,82 ; P=0,364\right)$. A type d'infestation égale, les fréquences des mono infestations ne diffèrent pas significativement $\left(X^{2}=0,57 ; P=0,452\right)$ entre les saisons. Par contre la prévalence des poly infestations est considérablement plus élevée $\left(X^{2}=4,82\right.$;
$P=0,028)$ en saison sèche qu'en saison pluvieuse. Lorsqu'on considère les catégories de poly infestations, il s'en suit l'existence de 4 associations parasitaires quelle que soit la saison. Les combinaisons penta spécifiques et hexa spécifiques sont exclusivement présentes en saison sèche et pluvieuse respectivement. Par ailleurs, l'occurrence des catégories de poly infestations diminuent très significativement $(P<0,001)$ lorsque le nombre de parasites associés augmente et quelle que soit la saison. Bien que les prévalences des catégories de poly infestations semblent être plus élevées en saison sèche, aucune différence significative n'est notée $(P>0,05)$.

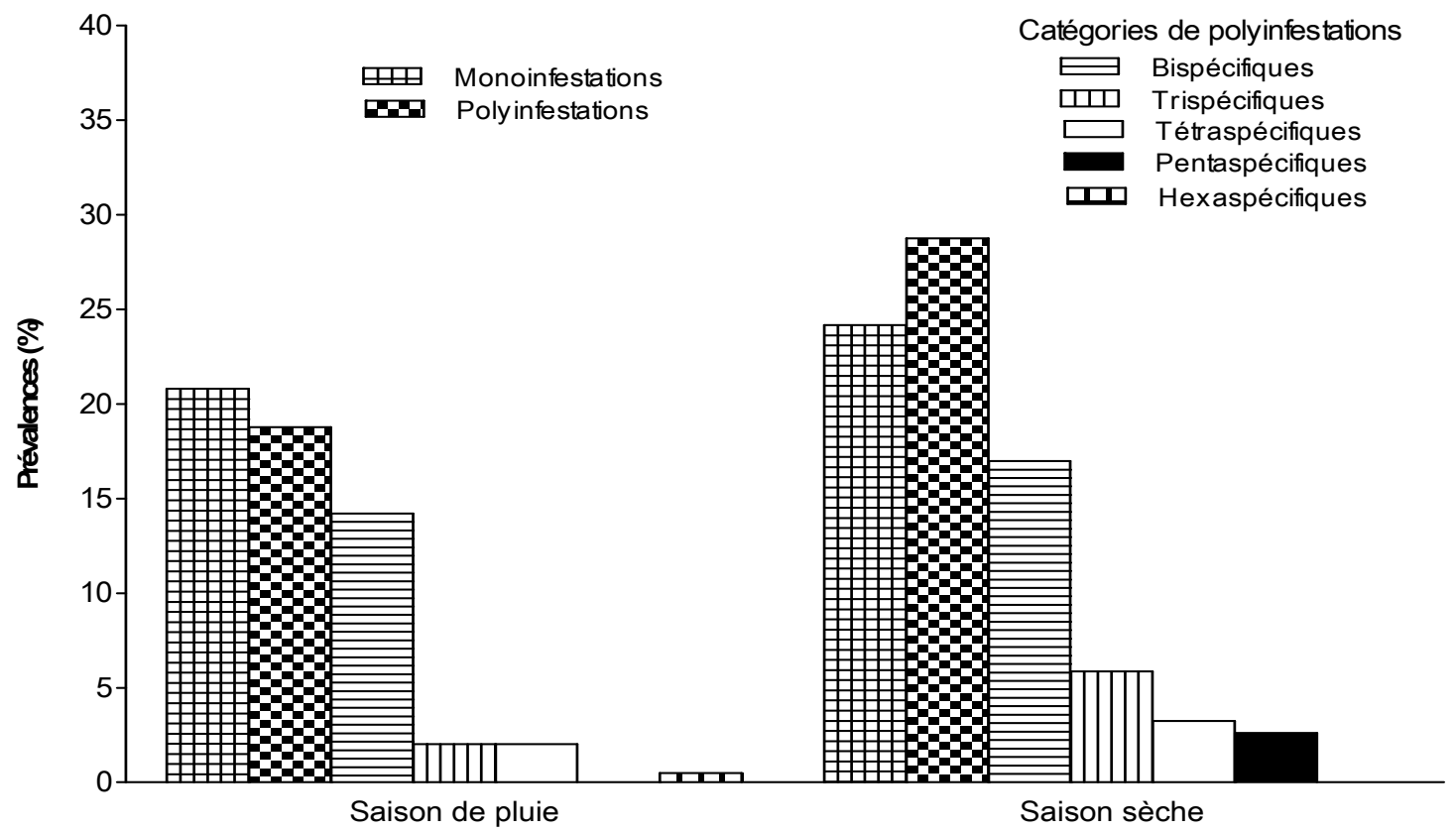

Figure 7 : Prévalences des types d'infestations en fonction des saisons

Prévalences des types d'infestations en fonction des classes de tailles: Les prévalences des types d'infestations en fonction des classes de tailles (Figure 8) montrent que les hôtes de toutes les classes de tailles considérées sont mono et polyinfestés. A type d'infestation égale, les prévalences des mono infestations augmentent $\left(X^{2}=0,49 ; P=0,781\right)$ avec la tailles des poissons. Pour ce qui est des poly infestations, la plus forte prévalence $\left(X^{2}=5,80 ; P=0,054\right)$ est enregistrée dans la classe] 100 - 150]. Quelle que soit la classe de taille, les prévalences des monoinfestations ne diffèrent pas significativement $(P>0,05)$ de celles des infestations multiples. Lorsqu'on prend en compte les catégories de polyinfestations, il apparaît que le nombre d'espèces associées baisse avec la taille du poisson. Ainsi, $5 ; 4$ et 3 catégories de polyinfestations sont observées respectivement dans les classes [50 - 100], ] 100 - 150] et $>150 \mathrm{~mm}$. La prévalence des infestations à 2 espèces est plus élevée $(P=0,001)$ que ce soit dans la première ou la deuxième classe de tailles. Les prévalences des catégories de poly infestations ne varient pas significativement entre les classes de tailles $(P>0,05)$. 


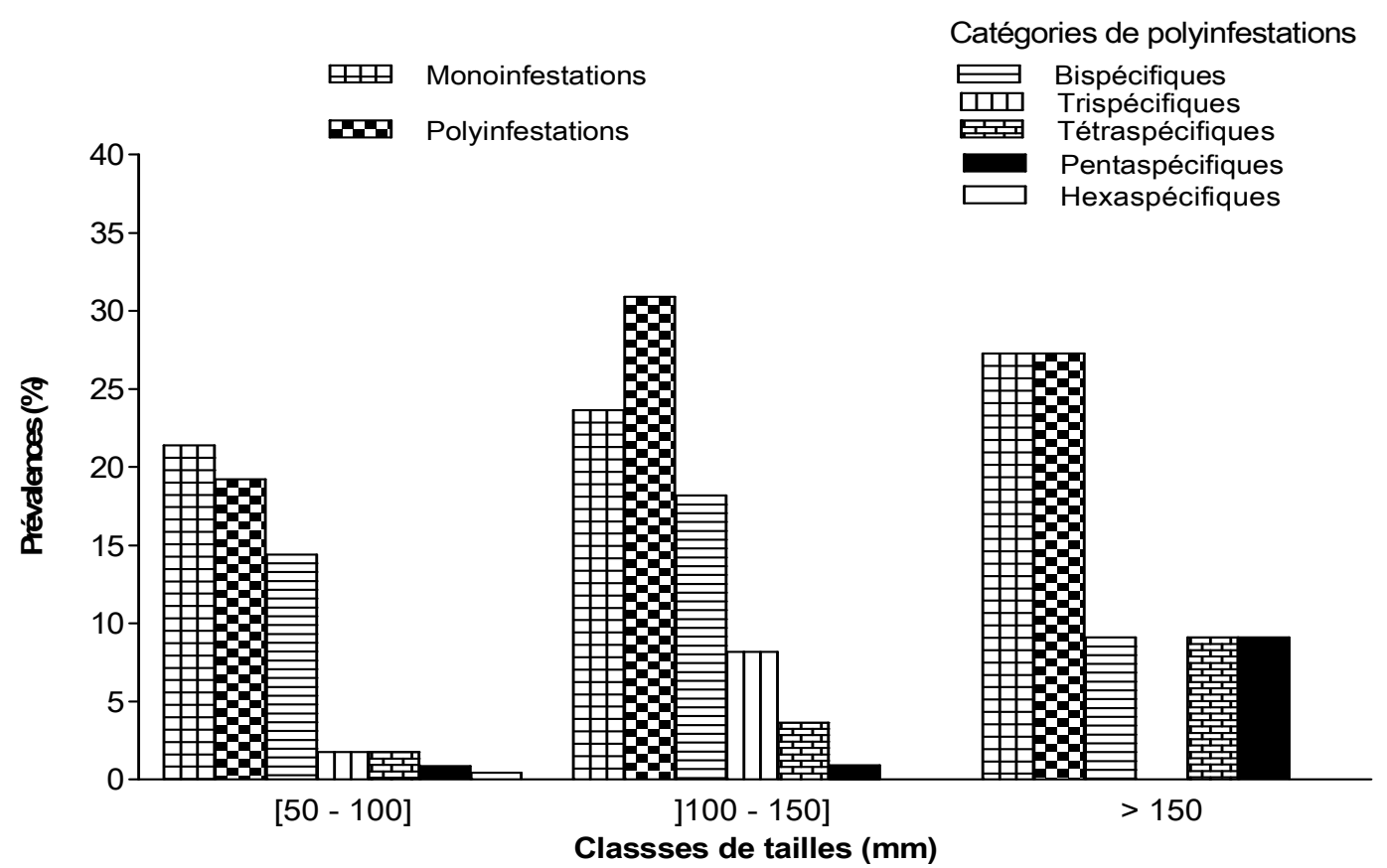

Figure 8 : Prévalences des types d'infestations en fonction des classes de tailles

\section{DISCUSSION}

La faune des myxosporidies inventoriée est riche et diversifiée, soit 12 espèces au total inféodées à 0 . niloticus. Nchoutpouen et al. (2011) ont recensé chez la même espèce de poisson dans la région administrative de l'Ouest-Cameroun 10 espèces de myxosporidies. Les faibles prévalences observées corroborent l'idée d'Euzet et Pariselle (1996) selon laquelle en milieu naturel, le taux de parasitisme est faible en raison de l'équilibre établi dans l'évolution du système hôte-parasite. Pour une espèce parasite donnée, le taux d'infestation des hôtes et le statut des parasites varient géographiquement (ElTantawi, 1989) et ce, en fonction des espèces hôtes (Brummer - Korvenkontio et al., 1991). Le polyparasitisme de nos poissons a été élucidé par Fomena (1996) à la station piscicole de Mélen-Cameroun et étaie la déclaration de Combes (1995) selon laquelle l'effet pathogène est rarement causé par une seule espèce parasite. La vulnérabilité de cet hôte serait liée aux facteurs endogènes tels que son comportement alimentaire. En effet ce poisson fouisseur se nourrit des algues sur lesquelles sont le plus souvent collées les actinospores. Uspenkaya, (1983) estime que les spores de myxosporidies sédimentent dans l'eau où elles vieillissent et deviennent mâtures dans la boue ou dans les vases pour pouvoir infester des nouveaux hôtes. Les poissons âgés ont été les plus parasités. La même observation a été faite par Nchoutpouen et al. (2011) en étangs. En effet la tendance est la diminution du taux d'infestation avec l'âge du poisson. Ainsi, Tombi et Bilong Bilong (2004), Viozzi et Flores (2003) puis, Abakar (2006) montrent que les jeunes poissons hôtes sont plus vulnérables aux myxosporidioses que ceux de grandes tailles. Ces auteurs expliquent ces observations par une augmentation de la réponse immunitaire avec l'âge du poisson. Les mêmes observations sont faites par Brummer - Korvenkontio et al. (1991) au centre de la Finlande. En effet, les prévalences de la myxosporidiose chez Rutilus rutilus par Myxobolus rhodei et $M$. pseudodispar tendent à diminuer quand l'âge du poisson augmente. Les prévalences ont été significativement plus élevées en saison sèche qu'en saison de pluie. Obiekezie et Okaeme (1990) pensent que les températures élevées de l'eau et la boue en saison sèche favoriseraient les infestations par les myxosporidies. Les oligochètes étant les hôtes intermédiaires dans le cycle de développement des myxosporidies (Markiw et Wolf, 1983), la variation saisonnière des myxosporidioses serait liée à la libération saisonnière des actinospores par les oligochètes (Özer et al., 2002). Le sexe du poisson n'a pas influencé sur les prévalences. La même observation a été faite par Abakar (2006), Milanin et al. (2010), puis Lekeufack et Fomena (2013). Fomena (1995) après ses travaux dans la station 

1758 (Cichlidae) au barrage réservoir de la Mapé (Adamaoua-Cameroun)

piscicole de Mélen (Yaoundé - Cameroun) ne trouve pas de différence entre les taux d'infestation des mâles et des femelles de Oreochromis niloticus par les myxosporidies des reins et de la rate. De même, Viozzi et Flores (2003) observent que la prévalence de Galaxias maculatus par Myxobolus biliare est indépendante du sexe de l'hôte et déclarent par conséquent qu'il s'agit d'une situation rencontrée dans les myxosporidioses. Les prévalences d'infestation des organes ont montré une différence significative. Les reins ont été significativement les plus parasités suivis du foie et de la rate. En effet, les reins jouent le rôle de filtration du sang et d'excrétion des solutés (Ellis et al.,1978), les parasites y convergeraient plus pour des besoins en métabolites divers. La spécificité organique ou la sélection des sites d'infestation serait liée à la préférence alimentaire du parasite et l'exclusion compétitive des autres espèces parasites. Le polyparasitisme d'un organe traduirait l'absence des compétitions entre les parasites (Sitjà-Bobadilla, 2008). Le large spectre d'infestation observé chez $M$. camerounensis s'expliquerait par une flexibilité (versatilité) des voies métaboliques. Ibrahim et Soliman (2010) pensent que l'hétérogénéité des biotopes crée des sites d'infestations distincts qui sont autant d'options d'habitats pour les parasites. La diminution des prévalences des catégories de polyinfestations avec l'augmentation du nombre d'espèces parasites associées pourrait s'expliquer par l'accroissement de l'intensité de la

\section{CONCLUSION ET APPLICATION DES RÉSULTATS}

Les prévalences des myxosporidioses chez 0 . niloticus ont été affectées par les facteurs endogènes (âge du poisson, sexe, organes) et exogènes (saisons). Les

\section{RÉFÉRENCES BIBLIOGRAPHIQUES}

Abakar O. (2006). Les myxosporidies (Myxozoa: Myxosporea) parasites des poissons D'eau douce du Tchad: Faunistique et biologie des espèces inféodées à Oreochromis niloticus (Linné, 1758) et Sarotherodon gallilaeus (Linné, 1758) cichlidae. Thèse de Doctorat d'État. Université de Yaoundé I.163p.

Baker J. R. (1963). Three new species of Myxosoma (Protozoa: Myxosporidia) from East African freshwater fish. Parasitology. 53: $285-292$.

Bilong Bilong C. F. et Jeannette Tombi (2004). Hétérogénéité du système branchial de Barbus martorelli roman, 1971 (poisson Cyprinidae) et modèle de croissance. Revue de l'académie des Sciences du Cameroun. Vol. 4. compétition interspécifique. Plus le nombre d'espèces associées est élevé, plus accentuée est la concurrence et moindres sont les prévalences d'infestations. Ce serait donc pour quoi l'association bispécifique présente une prévalence plus élevée par rapport à celles de la combinaison à 5 espèces parasites. Le parasitisme de nos poissons par les myxosporidies peut être à l'origine des plusieurs pathologies sévères. Myxobolus camerounensis provoquerait des lésions superficielles dans la peau ce qui constituent des portes d'entrée pour les pathogènes secondaires (Fomena, 1995). L'infection des yeux peut être cause de l'altération de la vision du poisson ce qui le prédispose à la prédation. Myxobolus heterosporus provoquerait chez des nombreux Cichlidae, des dégâts qui se répandraient dans le foie et la rate (Baker, 1963) et même dans l'intestin, ceci engendrera des septicémies, la perte des métabolites et le dysfonctionnement de l'osmorégulation. Ellis et al. (1978) soulignent que le rein des poissons téléostéens est un organe mixte ayant à la fois un rôle hématopoïétique, réticulo- endothélial, endocrine et excréteur. Ainsi, nous pensons que son infection peut créer des dysfonctionnements graves pour l'individu. Les branchies non seulement jouent un rôle respiratoire, mais aussi osmorégulateur. Son attaque peut être à l'origine de plusieurs perturbations. Obiekezie et Okaeme (1990) pensent que $M$. kainjiae détruirait les ovaires de 0 . niloticus d'où la baisse de sa fécondité.

données ainsi obtenues sont un pré requis à l'élaboration des stratégies de lutte contre les myxosporidioses afin de booster le potentiel de production en poissons.

Brummer - Korvenkontio, H. Valtonen E.T, Pugachev O. N. (1991). Myxosporea parasites in roach, Rutilus rutilus (Linnaeus) from four lakes in central Finland. J. Fish Biol., 38: 573 - 586.

Bush A.O., Lafferty K.D., Lotz J.M., Shostak A.W. (1997). Parasitology meets ecology on its own terms. J. Paraitol., 83: $575-583$.

Combes C. (1995). Interactions durables. Ecologie et évolution du parasitisme. Collection d'écologie, $\mathrm{n}^{\circ}$ 26. Paris. Ed. Masson : $524 \mathrm{P}$.

Ellis A.E., Robert R. J., Tytler P. (1978). The anatomy and physiology of teleost. In: Roberts R.J. Ed., Fish pathology. London, UK, Baillière Tindall, p.13-54.

El- Tantawi Sam (1989). Myxosporidian parasites fishes in lakes Dgal Weielki and warniak (Mazurian 
Lakeland, Poland). I. Survey of parasites. Acta parasitol. Polonica, 34 (3) : $203-219$.

Euzet L. et Pariselle A. (1996). Le parasitisme des poisons siluroidei: un danger pour l'aquaculture ? Aquatic Living resources. $9: 145$ $-151$.

Feist S.W. et Longshaw M. (2005). Myxozoan diseases of fish and effects on host population. Acta zool. Sin., 51(4): $758-760$.

FAO (2016). The state of world fisheries and aquaculture. Contributing to food security and nutrition for all. $24 \mathrm{p}$.

Fomena (1986). Contribution à l'étude des Myxosporidies (Protozoa : Myxozoa) parasites des Poissons d'eau douce du Sud- Cameroun : Systématique, Ultrastructure, relations hôte-parasite. Thèse de Troisième cycle. Université de Yaoundé. Cameroun. 276p.

Fomena A. (1995). Les Myxosporidies et Microsporidies des poissons d'eau douce du Sud - Cameroun : Étude faunistique, Ultrastructure et Biologie. Thèse de Doctorat d' État. Université de Yaoundé I. 397p.

Gbankoto A., Pampoulie C., Marques A. Sakiti G.N. ( 2001). Occurrence of myxosporean parasites in gills of tilapia species from Lake Nokoué (Benin, West Africa). Effect of host size and sex, and seasonal pattern of infection. Dis. Aqua. Organ., $44: 217$ - 222.

Ibrahim M.M. et Soliman F.M.M (2010). Prevalence and site preferences of heterophyid Metacercariae in Tilapia zilli from ismalia fresh water canal, Egypt. Parasite, 17: $233-239$.

Lekeufack Folefack G.B. et Fomena A. (2013). Structure et dynamique des infracommunautés de myxosporidies parasites de Ctenopoma petherici Günther, 1864 (Anabantidae), Clarias pachynema Boulenger, 1903 (Clariidae) et Hepsetus odoe (Bloch, 1794) (Hepsetidae) dans la rivière Sangé au Cameroun. Int. J. Biol. Chem. Sci.7(6) : $2301-2316$.

Lom J. et Arthur J.R. (1989). A guideline for the preparation of species description in Myxosporea. J. Fish. Dis., 12: 151 - 156.

Lom J. et Diková I. (2006). Myxozoan genera : definition and notes on taxonomy, life - cycle terminology and pathogenic species. Folia Parasitol., 53: 1 36.

Longshaw M., Freak P.A., Nunn A.D., Cowx I.G., Feist S.W., (2010). The influence of parasitism on fish population success. Fish. Manage. Ecol., 17: $246-434$.

Markiw M. E. et Wolf K. (1983). Myxosoma cerebralis (Myxozoa: Myxosporea) etiologic agent of Salmonid whirling disease requires tubificid worms (Annelida: Oligochaetea) in its life cycle. J. Protozool. 30: $561-564$

Milanin T., Eiras J.C., Arana S., Maia A.A.M., Alves A.L., Silva M.R.M., Carriero M.M., Ceccarelli, P.S. et Adriano E. (2010). Phylogeny, ultrastructure, histopathology and parasite of Brycon hilarii (Characidae) in the Pantanal Wetland, Brazil. Mem Inst Oswaldo Cruz, Rio de Janeiro, 105(6): 762-769.

MINEPIA (2015). Revue sectorielle Aquaculture Cameroun. Ministère de l'élevage, des pêches et des industries animales (MINEPIA), Yaoundé Cameroun. 44p.

Nchoutpouen E., Lekeufack Folefack G.B., Fomena A. (2011).Structure and population dynamics of Myxobolus infections in wild and cultured Oreochromis niloticus Linnaeus, 1758 in the Noun division (West- Cameroon). J. Cell Anim. Biol., 5: 245-264.

Obiekezie A. I. et Okaeme A. N. (1990). Myxosporea (Protozoa) infections of cultured tilapias in Nigeria. J. Afr. Zool.104: 77- 91.

Olivry J.C. (1986). Fleuves et rivières du Cameroun. O.R.S.T.O.M. (éd): 733p.

Özer A., Wootten R., Shinn A.P. (2002). Survey on actinosporean types (Myxozoa) belonging to seven collective groups found in a freshwater salmond in Northern Scotland. Folia parasitol., 49: $189-210$

Renault T. et Guichard B. (2007). Facteurs de risque d'apparition et d'émergence des maladies infectieuses en aquaculture. INRA prod. Anim., $20: 219-222$.

Sitjà - Bobadilla A. (2008). Fish immune response to Myxozoan parasites. Parasite, 15: 420 - 425.

Tombi, J. et Bilong Bilong, C.F. (2004). Distribution of gills parasites of the freshwater fish Barbus martorelli Roman, 1971 (Teleostei: Cyprinidae) and tendency to inverse intensity evolution Between Myxosporidia and Monogenea as a function of the host age. Revue Elev. réd. Vét. Pays trop. 57 (1-2): 71-76.

Uspenkaya A. V. (1995). Alternation of actinosporean and myxosporean phases in the life cycle of Zchokklella nova (Myxozoa). J. Eukaryot . Microbiol. 42: 665 - 668. 
Fonkwa et al., J. Appl. Biosci. 2018 Prévalences des Myxosporidioses Chez Oreochromis niloticus Linné, 1758 (Cichlidae) au barrage réservoir de la Mapé (Adamaoua-Cameroun)

Valtonen E.T., Holmes J.C., Koskivaara M. (1997).

Viozzi G. et Flores V. (2003). Myxidium biliare sp.n. ( Eutrophisation, pollution and fragmentation: Myxozoa) from gall bladder of Galaxias effects on parasite communities in roach (Ritilus rutilus) and perch (Perca fluviatilis) in four lakes in Central Finland. Can. J. Fish. Aqua. Sci., (54) : $572-585$. maculatus ( Osmeriformes: galaxidae) in patagonia (Argentina). Folia parasitologica. 50 : $190-194$ 